\title{
Methods available for improved anaesthetic outcome especially in the elderly
}

\author{
Keywords: blood, oxygen, mitochondrial, rebreathing
}

Abbreviations: MAP, mean arterial pressure; EEG, electroencephalographic; NIRS, near infra-red spectroscopy; POCD, post operative cognitive dysfunction

\section{Mini review}

I have been convinced that the tissues normally determine their own blood flow for at least 15 years. This has somehow not been adopted universally because of non-availability of free access to publications and partly because, it is such a general physiological point-affecting all organs in the body-that each specialist imagines, it is only really articles concerning advances in understanding their particular area that are likely to help them better understand things. Unfortunately this is totally erroneous as the amazing property of each organ is not only concerned just with blood flow but really precisely determining how fast oxygen is delivered to the particular tissue. Not just into the tissue as a whole but even specifically at the mitochondrial level. For example, when the optic area in a cat is monitored for neural activity, oxygenation and blood flow, flashing a light in the cat's eyes causes immediate increase in neural activity with proportional increase in local oxygen consumption and an increase in blood flow which is precisely three times the increase in oxygen consumption. There is an increase in $\mathrm{CO}_{2}$ production, though the increase in blood flow prevents a local rise in $\mathrm{PCO}_{2}$, which would have stimulated respiration. This also helps one understand that the increase in blood flow is not due to any increase in $\mathrm{PCO}_{2}$. Many authors in the past have assumed that an increase in arterial $\mathrm{PCO}_{2}$, for instance by hypoventilation or rebreathing of exhaled $\mathrm{CO}_{2}$, increases cerebral blood flow, as a primary mechanism. In contradiction to this, rise in $\mathrm{PCO}_{2}$ takes the $\mathrm{CO}_{2}$ regulating mechanism outside the normal range so the response is part of a 'broken' control mechanism. It is crucially important for the regulation of blood flow to the tissues to be intact for a normal blood volume. This is because Normal regulation of blood flow to the tissues requires an appropriate blood volume to sustain adequate pressures in the whole circulation.

The pressures in the whole circulation depend on the blood volume, but the arterial pressures, also depends on the relative compliance of the arteries and veins. If there is any change in compliance it will be in the venous side of the circulation, since arterial compliance, specifically related non-linearly to arterial blood pressure is otherwise invariant. Suddenly, it occurred to me and David Green ${ }^{1-3}$ that induction of anesthesia, was almost universally accompanied by a fall in arterial blood pressure and cardiac output with knowledge that there appeared to be a loss of circulating volume. The evidence for loss of volume was its improvement after an infusion of volume such as colloids or, with less persistent effect, crystalloids. So where had the volume gone when there was no blood loss? The answer was that the blood volume was unchanged but anesthesia had relaxed the venous system walls so blood had shifted from the arterial side of the circulation to the venous side. With the low pressure, blood flow was simply reduced in proportion to MAP (mean arterial pressure).
Volume I Issue 7 - 2017

\author{
Christopher B Wolff \\ William Harvey Research Institute, UK
}

Correspondence: Christopher B Wolff, Clinical Research Centre, William Harvey Research Institute, Barts and The London Hospital Medical and Dental School, Queen Mary College, Charterhouse Square, London ECIM 6BQ, UK, Email chriswolff@doctors.org.uk

Received: November 24, 2017 | Published: December 20, 2017

This is why the tissues became ischaemic. ${ }^{4}$ What was realised by Dr Green was that the venous relaxation could be countered by low doses of phenylephrine..$^{5}$ or later by Noradrenaline, with a return to normal pressure and blood flow (CO).

There are two other features of anesthesia which improve safety and enable avoidance of major circulatory problems and gross ischaemia. These are carefully explained in Dr Green's paper on Multi-Modal Monitoring. ${ }^{5}$ The first of these is the help provided by Electro-encephalographic (EEG) recording in determining the depth of anesthesia. There is a very great range of sensitivity to anesthetic agents in their effect on cerebral activity. If the anesthesia is too light the patient will be aware of what is going on, and could be in a position to sue. If too deep the danger of ischaemia from inadequate respiration of excess depression of cortical function is a major hazard.

A second system of protection is offered by monitoring cerebral function with Near Infra-red Spectroscopy (NIRS). This gives values of cerebral oxygenation, precisely what one needs to ensure emergence after anesthesia with an adequately oxygenated brain. Its value is from observation of any fall past a small routine reduction. With excess fall it is found that infusion of volume expander is required. If that doesn't work it is likely there has been haemorrhage, an early alert to find the source of bleeding. So, the requirements for anesthesia, safe from excess ischaemia, include observation of arterial blood pressure, adequacy of ventilation, measurement of cardiac output continuously as a component of multimodal monitoring, which includes monitoring cerebral function with both EEG and NIRS.

These methods are able to ensure a normal rate of oxygen delivery without which the major hazard is impairment of cognitive function. Unfortunately, impaired cognitive function is present postoperatively (post operative cognitive dysfunction, POCD) in a significant proportion of cases. The incidence is particularly high in the elderly. ${ }^{6}$ For patients over 60, over $40 \%$ had POCD on discharge from hospital and $12.7 \%$ still had a problem at 3 months? For cardiac surgery ${ }^{7}$ over $50 \%$ of patients had POCD at one week, with remission to still more than $30 \%$ at 2 months. Further evidence supporting cerebral ischaemia as the likely cause of POCD is the finding that for patients with severe bradycardia with cerebral dysfunction, it was relieved by introduction of a pacemaker. ${ }^{8}$ 
It is time for experienced anesthetists to ensure safer outcomes by using these methods (including starting monitoring prior to induction). This requires honest assessment of the evidence of the very great improvement brought about by the methods described here and $i^{5}$ and further papers, then pressing hard to ensure the equipment is available and used as suggested.

\section{Acknowledgements}

This work was facilitated by the NIHRB Biomedical Research Unit in Cardiovascular Disease at Barts.

\section{Conflict of interest}

The author declares no conflict of interest.

\section{References}

1. Wolff CB, Green DW. Clarification of the circulatory patho-physiology of anesthesia - Implications for high-risk surgical patients. Int J Surg. 2014;12(12):1348-1356
2. Wolff CB. Normal cardiac output, oxygen delivery and oxygen extraction. Adv Exp Med Biol. 2017;599:169-182.

3. Wolff CB, Collier DJ, Shah M, et al. A discussion on the regulation of blood flow and pressure. Adv Exp Med Biol. 2016;876:129-135.

4. Shoemaker WC, Appel PL, Kram HB. Role of oxygen debt in the development of organ failure, sepsis and death in high-risk surgical patients. Chest. 1992;102(1):208-215.

5. Green D, Bidd, Rashid H. Multimodal intraoperative monitoring: an observational case series in high risk patients undergoing major peripheral vascular surgery. Int J Surg. 2014;12(3):231-236.

6. Rundshagen I. Postoperative cognitive dysfunction. Dtsch Arztebl Int. 2014;111(8):119-125.

7. Bruggemans EK. Cognitive dysfunction after cardiac surgery: pathophysiological mechanisms and preventative strategies. Neth Heart J. 2013;21:70-73.

8. Koide H, Kobayashi S, Kitani M, et al. Improvement of cerebral blood flow and cognitive function following pacemaker implantation in patients with bradycardia. Gerontology. 1994;40(5):279-285. 